\title{
Congenital anomalies of the genitourinary system can help in diagnosis of the primary site of metastatic cancer: a case report and a review of the literature
}

\author{
This article was published in the following Dove Press journal: \\ OncoTargets and Therapy \\ 20 July 2016 \\ Number of times this article has been viewed
}

\author{
Andrzej Deptala ${ }^{1,2}$ \\ Agnieszka Romanowicz ${ }^{2}$ \\ Aleksandra Czerw ${ }^{3}$ \\ Jerzy Walecki ${ }^{4}$ \\ Wojciech Rogowski \\ Anna Nasierowska- \\ Guttmejer ${ }^{6}$ \\ 'Department of Oncology and \\ Hematology, Central Clinical \\ Hospital of the Ministry of Interior, \\ ${ }^{2}$ Department of Cancer Prevention, \\ ${ }^{3}$ Department of Public Health, Faculty \\ of Health Sciences, Medical University \\ of Warsaw, ${ }^{4}$ Department of Radiology, \\ Postgraduate Medical School, \\ ${ }^{5}$ Department of Urology, ${ }^{6}$ Department \\ of Pathology, Central Clinical Hospital \\ of the Ministry of Interior, Warsaw, \\ Poland
}

Objective: To analyze whether the presence of congenital anomalies of the genitourinary system that are accompanied by specific types of cancer and predispose patients to many complications, including infection, obstruction, stasis, calculus formation, and impaired renal function, could help in the diagnosis of the primary site of a metastatic tumor.

Case presentation: We report a case of a 58-year-old man with metastatic adenocarcinoma, in whom congenital anomalies of the genitourinary system proved helpful for the diagnosis of the primary site of cancer originating in the seminal vesicles.

Conclusion: We report an extremely rare case of primary adenocarcinoma arising probably from the left seminal vesicle associated with ipsilateral renal agenesis. The lesion was detected on ultrasound and contrast-enhanced computed tomography and confirmed histologically with ultrasound-guided biopsy. Serum markers, ie, CA19-9 and CA125, were elevated, while prostatespecific antigen and carcinoembryonic antigen were within normal limits. Such a constellation of markers strengthened the diagnosis. Our patient unfortunately presented very late in the course of the disease. Hence, we decided to initiate antiandrogen therapy and best supportive care in a hospice setting. Only early detection seems to be the key factor that may result in improved cure rates for cancer of the seminal vesicles. We also performed a literature search for current concepts related to the diagnosis and clinical management of primary adenocarcinoma of seminal vesicles.

Keywords: congenital anomalies of the genitourinary system, seminal vesicles carcinoma, case report, diagnosis, treatment

\section{Background}

Congenital anomalies of the genitourinary system (GUS) are very common and predispose patients to many complications, including infection, obstruction, stasis, calculus formation, and impaired renal function. Congenital GUS anomalies may be also accompanied by specific types of cancer, and therefore their presence could help in the diagnosis of the primary site of a metastatic tumor.

Primary cancers of seminal vesicles are extremely rare. ${ }^{1,2}$ Secondary involvement of seminal vesicles by prostate, bladder, and rectum cancers is much more frequent. ${ }^{1}$ In this report, we present the case of a patient with metastatic adenocarcinoma, in whom congenital anomalies of the GUS helped in the diagnosis of the primary site of a cancer originating in the seminal vesicles. We also performed a literature search for current concepts related to diagnosis and clinical management of primary adenocarcinoma of seminal vesicles.
Correspondence: Aleksandra Czerw Department of Public Health, Faculty of Health Sciences, Medical University of Warsaw, Zwirki i Wigury 6I, 02-091 Warsaw, Poland

Tel +4850I 176370

Email ola_czerw@wp.pl 


\section{Case presentation}

A 58-year-old man suffered from severe lower back pain, getting worse over the last 4 months, which affected his walking. He was admitted to the Central Clinical Hospital of the Ministry of Interior. He also complained of lack of appetite, weight loss ( $25 \mathrm{~kg}$ in 3 months), constipation, and increased urinary frequency. His previous medical history was irrelevant.

Physical examination revealed cachexia, Eastern Cooperative Oncology Group performance status 3, and a soft mass in the right iliac fossa and left umbilical region. In addition, a nontender tumor was palpable on digital rectal examination. Computed tomography (CT) of the abdomen and pelvis showed atrophic left kidney and normal right seminal vesicle (Figure 1A) and detected a large, multicystic mass measuring $125 \times 115 \times 110 \mathrm{~mm}$, growing from rectovesical excavation, compressing the bladder, sigmoid, and rectum (Figure 1B and C), probably arising from the left vesicle. Multiple bone metastases were present on visualized bones as well.

Laboratory tests showed anemia, thrombocytosis, hypercalcemia, and hematuria. Prostate-specific antigen (PSA) and carcinoembryonic antigen (CEA) levels were normal. CA19-9 was slightly elevated at $52.1 \mathrm{IU} / \mathrm{mL}$ (normal range $0-34 \mathrm{IU} / \mathrm{mL}$ ) and so was CA125 at $36.1 \mathrm{IU} / \mathrm{mL}$ (normal range 0-35 IU/mL). Chest X-radiograph disclosed multiple round opacities consistent with metastases. Sigmoidoscopy revealed a slightly narrowed lumen of the sigmoid and rectum, caused by external compression, but otherwise was normal. Ultrasound-guided transrectal biopsy of the tumor was performed. The histopathological evaluation (Figure 2A and B) confirmed moderately differentiated adenocarcinoma of the seminal vesicles.

Owing to the disseminated stage of the disease and the patient's poor performance status, antiandrogen therapy with flutamide was offered together with supportive care.
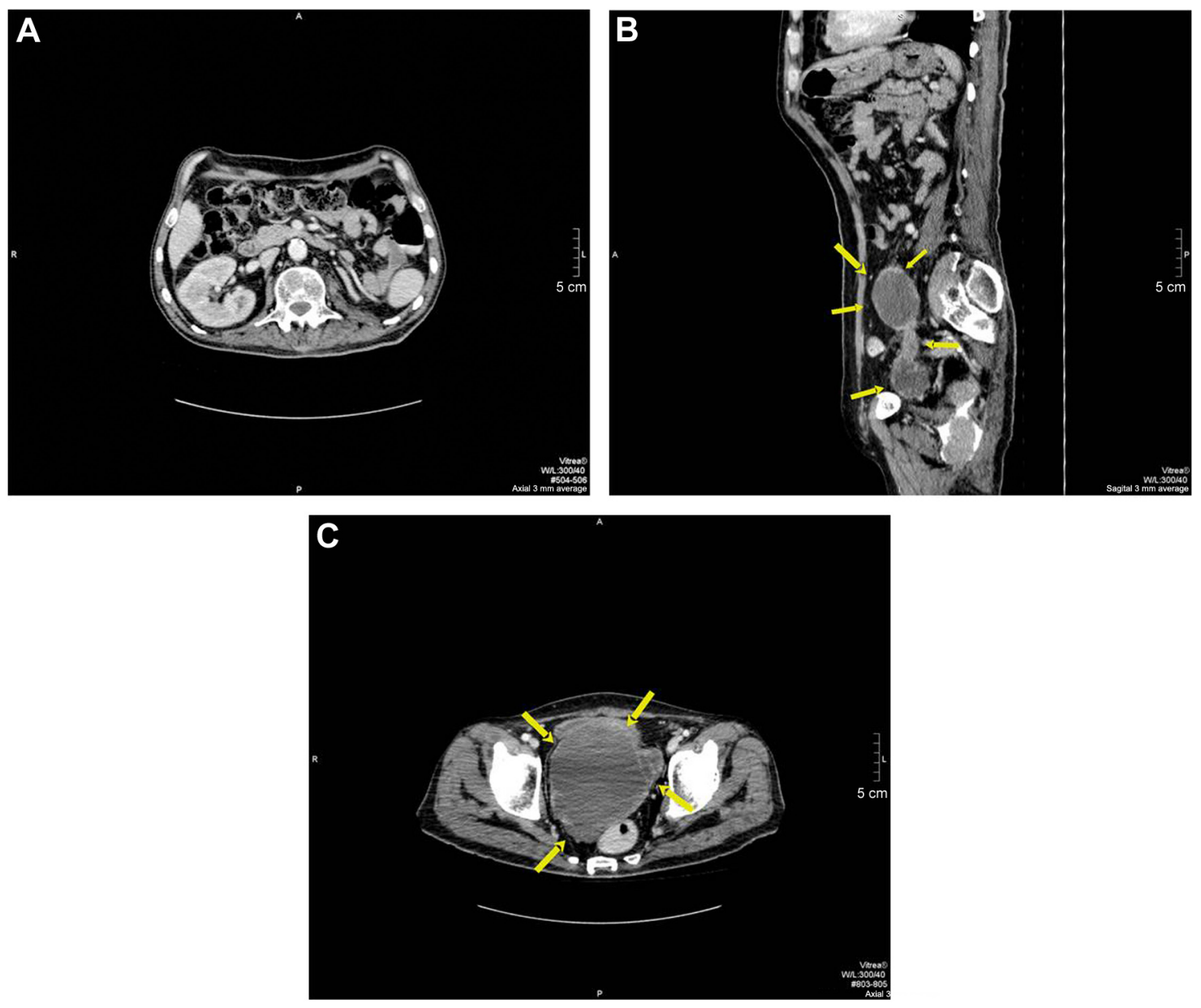

Figure I Contrast-enhanced CT.

Notes: (A) Atrophic or dysgenetic left kidney. (B and C) Papillary solid mass (arrows) growing from rectovesical excavation, compressing the bladder, sigmoid, and rectum. Abbreviation: CT, computed tomography. 

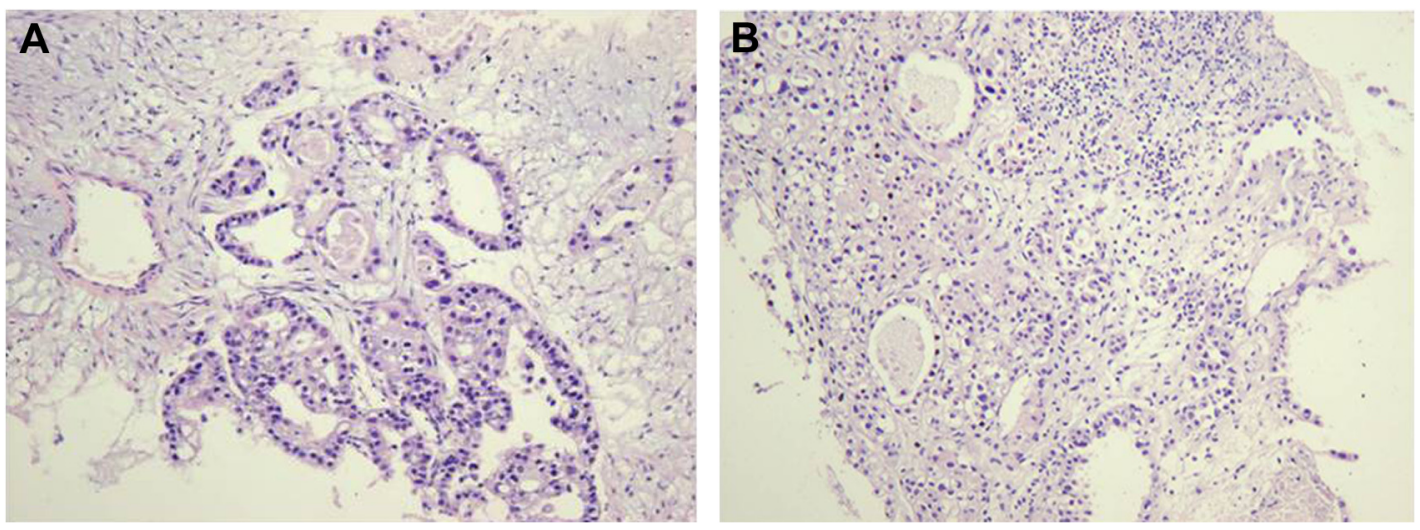

Figure 2 Adenocarcinoma of the seminal vesicles.

Notes: (A) Moderately differentiated adenocarcinoma of the seminal vesicles forming tubular structures and solid infiltration in the stroma. (B) Mature adenocarcinoma of the seminal vesicle. Magnification $\times 100$.

However, the patient passed away in a few weeks following beginning of the treatment.

\section{Consent}

Since the patient had died, the consent for publication was signed by the patient's wife.

\section{Literature review Etiology}

Primary tumors of seminal vesicles are extremely rare. ${ }^{1,2}$ Cystadenomas and mesenchymal tumors are benign primary lesions of the seminal vesicles, while the malignant ones are sarcomas, chorionepitheliomas, and the most common adenocarcinomas. ${ }^{2-4}$ The first case of carcinoma of seminal vesicles was reported by Lyons in 1925.,5 Since then, only 50 documented cases have been described in patients aged $19-90$ years. $^{2}$

Etiology of primary seminal vesicle adenocarcinoma remains unclear. Besides the androgens influence in the development of that carcinoma, several authors report the role of some congenital anomalies of the GUS. In 2007, Lee et $\mathrm{al}^{1}$ reported a case of primary mucinous adenocarcinoma arising from a seminal vesicle cyst that was associated with an ectopic ureter and ipsilateral renal agenesis. ${ }^{1}$ A similar case of papillary adenocarcinoma of the seminal vesicle in a patient with ipsilateral renal agenesis but without concomitant ectopic ureter was published in 1992 by Okada et al. ${ }^{6}$ Urethral ectopia more often develops in females than in males. ${ }^{1,7}$ This abnormality in males usually involves drainage into the seminal vesicle and may also be associated with reflux or stricture that may affect renal function or cause its agenesis. ${ }^{1,8}$ Congenital seminal vesicle cysts are commonly associated with renal agenesis or dysgenesis on the ipsilateral side..$^{1,9}$
The incomplete development of Wolffian duct and the urogenital sinus in males result in an accumulation of secretions and the subsequent formation of seminal vesicle cysts during puberty. ${ }^{1,10}$ Lee et al in their review suggested that the development of seminal vesicle adenocarcinoma may be due to chronic stimulation of seminal vesicle by secretions from the ectopic ureter. In our patient with the primary seminal vesicle, adenocarcinoma agenesis of the kidney was detected; however, ectopia of the ureter could not be confirmed due to the very advanced stage of the disease and the extent of malignant infiltration at the time of diagnosis. For the same reason, we were unable to confirm for sure which seminal vesicle was the primary site of the adenocarcinoma.

\section{Diagnosis and clinical symptoms}

Primary seminal vesicle carcinoma occurs in adult males regardless of age, and in contrast to prostate carcinoma, the morbidity rate does not rise with age.

The diagnostic criteria for cancer of the seminal vesicles proposed by Dalgaard and Giertsen in 1956 are continually being applied and include primary focus of carcinoma in the seminal vesicles and absence of concomitant lesion in the prostate.

The tumor's architecture should preferably resemble the normal tissue of the seminal vesicle. ${ }^{11-13}$

These criteria can be easily applied to resected specimens, but they are less valuable in relation to a biopsy material; ${ }^{12,13}$ however, they have been met in our case.

Early diagnosis of seminal vesicle carcinoma is difficult because clinical signs and symptoms are usually absent. When symptoms occur, they often reflect either a locally advanced disease with infiltration of adjacent organs (bladder, rectum and prostate) or a metastatic spread. ${ }^{13,14}$ Frank hematuria and 
hematospermia are the most common symptoms of locally advanced seminal vesicle carcinoma causing infiltration and obstruction of the bladder outlet. ${ }^{2,13,14}$ Patients also complain of dysuria, pelvic pain, or pain on defecation. ${ }^{2,13,14}$

Digital rectal examination is essential as it allows detection of a nontender tumor above prostate. However, cases without abnormalities on digital examination or cystoscopy have also been reported in the literature. ${ }^{2,15-17}$ In these patients, lesions were detected incidentally at transurethral resection of the prostate or during an autopsy. ${ }^{2}$

\section{Diagnostic tests}

Historically, vesiculography was used to assess the pathology of the seminal vesicles. Owing to its invasive nature and availability of $\mathrm{CT}$ and magnetic resonance imaging, vesiculography has generally been abandoned in recent years. ${ }^{2}$ Imaging with increased sensitivity for abnormalities of the seminal vesicles has become available with the introduction of transrectal ultrasound and $\mathrm{CT} .{ }^{2}$ A retrovesical or seminal vesicle mass was detectable in almost all the patients with seminal vesicle carcinoma undergoing pelvic CT. ${ }^{2,18-20}$ Prostate cancer should be excluded in each case by digital rectal examination, PSA measurement, and transrectal ultrasound, as was done in our patient. Sigmoidoscopy is recommended to rule out rectal carcinoma and contributes to local staging of seminal vesicle cancer.

Owing to the rarity of the disease, TNM classification has not been established for this disease yet. ${ }^{2}$ Urine or semen cytology is not recommended in the diagnosis due to lack of specificity. However, serum analysis of PSA, CEA, and most importantly, CA125 has been especially helpful in the differential diagnosis., ${ }^{2,21}$ Normal levels of PSA and CEA could suggest the absence of secondary invasion of the seminal vesicles by prostate or colon carcinoma. ${ }^{2}$ High levels of CA125 are strongly associated with this cancer. ${ }^{2,22}$ Furthermore, it has been proven that the CA125 level correlates with the tumor's size and disease recurrence and is useful in the assessment of resectability. ${ }^{2,22}$ It is suggested in the literature that CA125 level can be used as a serological marker to monitor treatment response.

The most important factor in the diagnostic workup of seminal vesicles carcinoma is histopathological evaluation. Specimens for pathological assessment could be obtained by transrectal tumor biopsy - with or without US guidance - or surgical excision of the lesion localized in the pelvis. In some patients, the diagnosis was based on histopathological assessment of the tissue removed by transurethral resection of the prostate or bladder neck, performed for hematuria or bladder outlet obstruction symptoms. Immunohistochemistry of the pathology specimen is more helpful to exclude metastatic tumors than to confirm the origin of the tumor in the seminal vesicle. ${ }^{13}$ In cases reported up to date in the literature, the primary seminal vesicle carcinoma is negative for PSA and also for antiprostate-specific acid phosphatase. ${ }^{13}$ As the seminal vesicles could often be invaded by prostatic adenocarcinoma, the PSA negativity in the immunostaining is very useful in differential diagnosis between primary and metastatic infiltration, but does not completely exclude anaplastic prostatic adenocarcinoma, which may be PSA negative. ${ }^{13,20}$ Primary seminal vesicle carcinoma usually expresses CK7 but is negative for CK20. Lack of CK20 expression may distinguish seminal vesicle carcinoma from colon adenocarcinoma and urothelial-type bladder carcinoma, which usually are CK20 positive. Most of the well-differentiated seminal vesicle adenocarcinomas are CA125 positive, while poorly differentiated cancers do not express this marker. ${ }^{13,16,20}$

\section{Treatment}

There is no established standard of care in primary seminal vesicle carcinoma due to its extremely rare incidence. In reported cases, treatment strategies have primarily concentrated on attempts to excise the tumor surgically. ${ }^{2}$ The extent of resection is dependent on the degree of involvement of adjacent organs by the seminal vesicle tumor as well as the patient age. Surgical approaches have ranged from local excision of a seminal vesicle to more aggressive surgeries, including retropubic prostatectomy-vesiculectomy, cystoscopy-prostatectomyvesiculectomy with urinary diversion, and even pelvic exenteration. ${ }^{2}$ Radical prostatectomy-vesiculectomy in the seminal vesicle carcinoma is reasonable if prostate infiltration is suspected because of the wide use and experience of radical prostatectomy in patients with prostate cancer. ${ }^{2}$ Only tumors without prostate involvement may be treated by vesiculectomy alone. $^{2}$

Radiotherapy has been used as an adjuvant treatment modality in the seminal vesicle carcinoma following surgery, especially when the surgical margins were positive..$^{2,5,15,20}$ According to the reported data, chemotherapy was not used in the adjuvant setting. In the metastatic settings, treatment with regimen containing fluorouracil (modulated with folinic acid) and oxaliplatin can be offered to patients with a good performance status. ${ }^{5,22,23}$ However, therapeutic results of this approach are very disappointing.

More data are available regarding the benefits of hormonal therapy in the treatment of seminal vesicle carcinoma in both adjuvant and palliative settings.,23,24 The rationale for 
antiandrogen therapy or bilateral orchidectomy is derived from the androgen-dependent development and function of the seminal vesicles. The existing evidence indicates that hormonal manipulation may prolong overall survival in few cases over 24 months. Treating with a combination of chemotherapy plus antiandrogen therapy allows a relapse-free survival for $>16$ months. ${ }^{23}$

The long-term prognosis for patients with seminal vesicle carcinoma is extremely poor mainly due to diagnosis in advanced or metastatic state. ${ }^{2,11,18,24}$ However, in many reported cases, survival or recurrence rates have not been provided and follow-up has been too short.

\section{Conclusion}

We report here an extremely rare case of primary adenocarcinoma arising from the seminal vesicle (probably left) associated with ipsilateral renal agenesis. Owing to dissemination of the carcinoma, it was not possible to prove which seminal vesicle was the primary site. The lesion was detected on ultrasound and contrast-enhanced CT and confirmed histologically with ultrasound-guided biopsy. Serum markers, ie, CA19-9 and CA125, were elevated, while PSA and CEA were within normal limits. Such a constellation of markers strengthened the diagnosis. Our patient unfortunately presented very late in the course of the disease. Hence, we decided to initiate antiandrogen therapy and best supportive care in a hospice setting. Our second conclusion aims to emphasize that only early detection seems to be the key factor that may result in improved cure rates for cancer of the seminal vesicles.

\section{Author contributions}

All authors made substantial contributions to conception and design, acquisition of data, analysis and interpretation of data, drafting and critically revising the paper, gave final approval of the version to be published, and agree to be accountable for all aspects of the work.

\section{Disclosure}

The authors report no conflicts of interest in this work.

\section{References}

1. Lee BH, Seo JW, Han YH, Kim YH, Cha SJ. Primary mucinous adenocarcinoma of a seminal vesicle cyst associated with ectopic ureter and ipsilateral renal agenesis: a case report. Korean J Radiol. 2007;8(3): 258-261.
2. Thiel R, Effert P. Primary adenocarcinoma of the seminal vesicles. J Urol. 2002;168(5):1891-1896.

3. Hajdu SI, Faraque AA. Adenocarcinoma of the seminal vesicle. J Urol. 1968;99(6):798.

4. Lyons O. Primary carcinoma of left seminal vesicle. J Urol. 1925; 13:477-478.

5. Yuvaraja BT, Hemant BT, Sudeep G, Sumeet G. Primary seminal vesicle adenocarcinoma presenting as isolated metastasis to penis responding to chemotherapy and hormonal therapy. Urology. 2007;69(4): 778.e1-e3.

6. Okada Y, Tanaka H, Takeuchi H, Yoshida O. Papillary adenocarcinoma in a seminal vesicle cyst associated with ipsilateral renal agenesis: a case report. J Urol. 1992;148(5):1543-1545.

7. Matsuki M, Matsuo M, Kaji Y, Okada N. Ectopic ureter draining into seminal vesicle cyst: usefulness of MRI. Radiat Med. 1998;16(4): 309-311.

8. Levisay GL, Holder J, Weigel JW. Ureteral ectopia associated with seminal vesicle cyst and ipsilateral renal agenesis. Radiology. 1975;114(3): 575-576.

9. Heaney JA, Pfister RC, Meares EM. Giant cyst of the seminal vesicle with renal agenesis. AJR Am J Roentgenol. 1987;149(1):139-140.

10. Zaontz MR, Kass EJ. Ectopic ureter opening into seminal vesicle cyst associated with ipsilateral renal agenesis. Urology. 1987;29(5): 523-525.

11. Dalgaard JB, Giertsen JC. Primary carcinoma of the seminal vesicle: case and survey. Acta Pathol Microbiol Scand. 1956;39(4):255-267.

12. Ormsby AH, Haskell R, Jones D, Goldblum JR. Primary seminal vesicle carcinoma: an immunohistochemical analysis of four cases. Mod Pathol. 2000;13(1):46-51

13. Tarjan M, Ottlecz I, Tot T. Primary adenocarcinoma of the seminal vesicle. Indian J Urol. 2009;25(1):143-145.

14. Egevad L, Ehrnström R, Håkansson U, Grabe M. Primary seminal vesicle carcinoma detected at transurethral resection of prostate. Urology. 2007;69(4): 778.e11-e13.

15. Davis NS, Merguerian PA, DiMarco PL, Dvoretsky PM, Rashid H. Primary adenocarcinoma of the seminal vesicle presenting as bladder tumor. Urology. 1988;32(5):466-468.

16. Ormsby AH, Haskell R, Ruthven SE, Mylne GE. Bilateral primary seminal vesicle carcinoma. Pathology. 1996;28(2):196-200.

17. Steininger H. Primares Samenblasenkarzinom [Primary seminal vesicles carcinoma]. Pathologe. 1987;8(3):183. Dutch.

18. Benson RC, Clark WR, Farrow GM. Carcinoma of the seminal vesicle. J Urol. 1984;132(2):483-486.

19. Oguchi K, Takeuchi T, Kuriyama M, Tanaka T. Primary carcinoma of the seminal vesicle (Cross-imaging diagnosis). Br J Urol. 1988;62(4): 383-384.

20. Oxley JD, Brett MT, Gillatt DA, Burton P. Seminal vesicle carcinoma. Histopathology. 1999;34(6):562-563.

21. Lathem JE. Carcinoma of the seminal vesicle. South Med J. 1975; 68(4):473.

22. Ohmori T, Okada K, Tabei R, et al. CA125-producing adenocarcinoma of the seminal vesicle. Pathol Int. 1994;44(4):333-337.

23. Rodriguezkees OS. Clinical improvement following estrogenic therapy in a case of primary adenocarcinoma of the seminal vesicle. JUrol. 1964;91: 665-670.

24. Awadalla O, Hunt AC, Miller A. Primary carcinoma of the seminal vesicle. Br J Urol. 1968;40(5):574-579. 


\section{Publish your work in this journal}

OncoTargets and Therapy is an international, peer-reviewed, open access journal focusing on the pathological basis of all cancers, potential targets for therapy and treatment protocols employed to improve the management of cancer patients. The journal also focuses on the impact of management programs and new therapeutic agents and protocols on

patient perspectives such as quality of life, adherence and satisfaction. The manuscript management system is completely online and includes a very quick and fair peer-review system, which is all easy to use. Visit http://www.dovepress.com/testimonials.php to read real quotes from published authors.

Submit your manuscript here: http://www.dovepress.com/oncotargets-and-therapy-journal 